\section{El control de la calidad de los análisis inmunohematológicos en la Región de las Américas}

\author{
Elena Franco ${ }^{1}$
}

Palabras clave: control de calidad, inmunohematología, transfusión sanguínea.

\footnotetext{
1 Centro de Transfusión de la Comunidad Valenciana, Valencia, España. Dirección postal: Avda. del Cid, 65 acc. Valencia 46014, España. Fax: +34 96 3868109. Correo electrónico: franco_ele@gva.es
}

Si bien conseguir la excelencia es meta de todas las especialidades médicas, la medicina transfusional ha sido una de las que más medidas ha adoptado en los últimos años para lograrla. Ello se debe a que esta especialidad se sitúa en el punto de mira de la sociedad en general y a que las transfusiones sanguíneas siguen presentando riesgos, aunque sean mínimos, para la salud de los receptores. Desde que comenzó la pandemia de sida a mediados de los años ochenta, muchos aspectos de la práctica de la especialidad han sido sometidos a un control exhaustivo.

La creciente demanda de calidad y seguridad por parte de los pacientes y de la sociedad en general en relación con el tratamiento transfusional ha impulsado esfuerzos continuos para mejorar las prácticas y garantizar que la transfusión cumpla con determinados objetivos. Tales esfuerzos han conducido a notables logros, ya que se han introducido medidas de seguridad más rigurosas en cada paso intermedio desde la selección del donante hasta el seguimiento postransfusional del paciente.

La mayoría de los países han adoptado leyes aplicables a la terapia transfusional, tanto en sus aspectos organizativos como en los eminentemente técnicos, para garantizar la seguridad de la transfusión sanguínea. Por esta razón, se está superando el concepto de control de calidad y actualmente se habla de la garantía de la calidad, habida cuenta de la necesidad de ejercer un estricto control sobre todo el sistema y de establecer y observar protocolos para todos los pasos, desde la obtención de la sangre del donante hasta su administración al receptor.

La correcta tipificación de las muestras sanguíneas y la detección de anticuerpos irregulares en todas las donaciones de sangre constituyen una parte crucial del proceso de garantizar que la transfusión cumpla con sus objetivos terapéuticos sin provocar efectos indeseados, algunos de los cuales podrían poner en riesgo la vida del paciente. Por lo tanto, la obtención de resultados correctos en las técnicas inmunohematológicas aplicadas de forma generalizada para constatar la perfecta caracterización de las unidades de sangre o de sus componentes es fundamental para garantizar una buena asistencia transfusional.

Son varios los aspectos que deben controlarse para conseguir resultados correctos. En esta búsqueda de la calidad desempeñan un papel determinante factores tales como los reactivos utilizados, la 
precisión de los instrumentos y equipos, las técnicas utilizadas y, desde luego, la formación y destreza del personal que las ejecuta. Al mismo tiempo, es preciso contar con una organización que impida, o al menos reduzca a un mínimo, el error humano en cualquiera de los puntos de la cadena transfusional una vez que estén reguladas mediante un protocolo todas las medidas que inciden en la calidad, desde la procedencia de las muestras hasta la identificación correcta del receptor, pasando por la transcripción de los resultados analíticos a los registros, bien sean electrónicos o manuales. En este artículo se examinan más ampliamente todos estos aspectos.

Cada vez son más numerosas las organizaciones e instituciones que se adhieren de forma voluntaria a programas de control de calidad externos para comprobar de forma periódica la fiabilidad de los resultados de laboratorio que obtienen, hecho que demuestra sin duda su interés y compromiso con la calidad asistencial. La Organización Panamericana de la Salud (OPS), consciente de esta necesidad, apoya desde 1996 el Programa de Control de Calidad Externo en Inmunohematología para la Región de las Américas, que está proporcionando muy buenos resultados.

\section{MECANISMOS PARA ELIMINAR ERRORES}

Cometer errores es parte consustancial de la condición humana, pero a su vez es algo que en la práctica médica no se puede permitir. Por esta razón, la organización y los métodos de trabajo de cualquier centro deben tener como objetivo establecer barreras contra posibles fuentes de error. Toda organización debe recoger y analizar los errores observados, así como los que estuvieron a punto de cometerse, porque de su estudio deben nacer las medidas encaminadas a evitar que se repitan. Las causas de error más frecuentes en un laboratorio de inmunohematología suelen ser de origen organizativo o técnico:

\section{Errores de origen organizativo}

Identificación incorrecta de las muestras. Puede no estar indicado el origen del tubo de sangre piloto o el de la unidad de sangre correspondiente, o bien el del tubo piloto y la identidad del paciente al que se le debe transfundir la sangre. Para evitar errores de identificación se debe usar de modo rutinario un sistema numérico que debe ser bien conocido por todos los miembros de la organización encargados de ejecutar tareas transfusionales, sea o no informa- tizado el sistema. En el caso de las unidades de sangre, siempre se deberá proceder a analizar los grupos $\mathrm{ABO}$ y $\mathrm{D}$ por duplicado, una vez con la sangre del tubo que acompaña a la bolsa de sangre y otra con el tubo piloto, anotando cuidadosamente la identidad de ambos.

Errores en la transcripción de los resultados a los registros, sean estos informáticos o manuales. En el caso de registros informáticos se evitan los errores en los procesos que se realizan automáticamente mediante conexiones en línea. Cuando la transcripción es manual, el técnico que hace el análisis y transcribe los resultados está obligado a firmar y su firma sirve como comprobante. Aunque la existencia de protocolos obliga a todos los técnicos a realizar las pruebas de una misma manera, no se recomienda que una persona inicie una técnica y que otra la termine y registre el resultado.

\section{Errores de origen técnico}

Los reactivos. Los problemas pueden deberse, por ejemplo, a la caducidad de los reactivos o a su alteración por almacenamiento en condiciones poco idóneas. Es necesario realizar un control de la reactividad de los mismos con una o más muestras conocidas.

Las muestras. La calidad de las muestras puede verse afectada por las técnicas de extracción y de conservación.

El equipo. Los instrumentos empleados pueden ser defectuosos.

Los métodos. Pueden surgir errores debido, entre otras cosas, a prácticas inadecuadas o a no seguir las instrucciones del fabricante de los reactivos usados.

El uso sistemático de controles conocidos en conexión con todas las técnicas aplicadas alertará sobre cualquier problema técnico. Además, es conveniente recordar aquí la enorme importancia de determinar siempre el grupo hemático y sérico al que pertenece toda muestra como mecanismo para garantizar la correcta identificación del grupo ABO.

\section{LA CALIDAD DE LOS REACTIVOS}

Todas los centros adquieren los reactivos que van a utilizar después de comprobar que cumplen sus expectativas técnicas. Ello no significa, no obstante, que no deban someterse a controles sistemáticos, que deben abarcar: 
CUADRO 1. Control de la calidad de los reactivos. Solución de baja fuerza iónica (LISS)

\begin{tabular}{lll}
\hline $\begin{array}{l}\text { Parámetros que } \\
\text { se deben controlar }\end{array}$ & \multicolumn{1}{c}{ Requisitos cualitativos } & Frecuencia de los controles \\
\hline Apariencia & Ausencia de turbidez o partículas detectables por examen visual & Diaria \\
$\mathrm{pH}$ & $6,7(6,5 \mathrm{a} 7)$ & Cada nuevo lote \\
Conductividad & $3,7 \mathrm{mS} / \mathrm{cm}(3,44$ a 3,75$)$ a $23^{\circ} \mathrm{C}$ & Cada nuevo lote \\
\hline
\end{tabular}

CUADRO 2. Control de la calidad de los reactivos. Hematíes

\begin{tabular}{lll}
\hline $\begin{array}{l}\text { Parámetros que } \\
\text { se deben controlar }\end{array}$ & Requisitos cualitativos & Frecuencia de los controles \\
\hline Apariencia & $\begin{array}{c}\text { Ausencia de turbidez o hemólisis en el sobrenadante por examen } \\
\text { visual } \\
\text { Reacciones claras con sueros seleccionados frente a los antígenos } \\
\text { eritrocitarios }\end{array}$ & $\begin{array}{c}\text { Cada nuevo lote, el primero } \\
\text { yeactividimo día de vida }\end{array}$ \\
\hline
\end{tabular}

CUADRO 3. Control de la calidad. Proteasas

\begin{tabular}{llc}
\hline $\begin{array}{c}\text { Parámetros que } \\
\text { se deben controlar }\end{array}$ & Requisitos cualitativos & Frecuencia de los controles \\
\hline Reactividad & $\begin{array}{l}\text { Ausencia de aglutinación o hemólisis empleando un suero AB inerte } \\
\text { Aglutinación de los hematíes sensibilizados con un anticuerpo anti-D } \\
\text { de tipo lgG a título débil }\end{array}$ & Cada nuevo lote \\
Potencia & $\begin{array}{l}\text { Un suero de tipo lgG, preferiblemente anti-D, estandarizado en } \\
\text { un título de } 1 / 64 \text { a } 1 / 128 \text { debe dar esta misma titulación con los } \\
\text { diferentes lotes de la enzima }\end{array}$ & Cada nuevo lote \\
\hline
\end{tabular}

1. El registro, en el momento de la recepción de los reactivos, de que las condiciones de embalaje, temperatura y caducidad son adecuadas.

2. La evaluación de cada lote en el laboratorio antes de usarlo (cuadros 1 a 6 ).

3. El control de las condiciones de almacenamiento.

4. El cumplimiento de las instrucciones del fabricante para garantizar resultados correctos.

5. El análisis diario de controles internos apropiados para garantizar la corrección y repetibilidad de los resultados.

Cualquier error o problema debe ser convenientemente registrado y comunicado.

\section{LA CALIDAD DE LAS TÉCNICAS}

Todos los procedimientos de trabajo deben estar escritos con claridad y concisión y, una vez que estén aprobados, deberán colocarse en un lugar de fácil acceso para que el personal pueda consultarlos. Nadie puede practicar las técnicas de una forma distinta de la aprobada y no hay que olvidar que la mayoría de los errores que se cometen se pueden evitar si se siguen las normas. Es preciso:

1. Identificar y ordenar las muestras correctamente.

2. Controlar los reactivos.

3. Estudiar controles positivos y negativos junto con las muestras problemáticas y constatar que se obtienen los resultados esperados (cuadros 7 y 8).

4. Establecer un sistema a prueba de errores para el registro de los resultados.

\section{LA CALIDAD DE LOS INSTRUMENTOS}

El equipo que contiene un laboratorio debe ser el adecuado para el uso al que está destinado. Para garantizar su correcto funcionamiento es necesario cumplir las siguientes normas: 
CUADRO 4. Control de calidad de los reactivos. Sueros ABO

\begin{tabular}{|c|c|c|}
\hline $\begin{array}{l}\text { Parámetros que } \\
\text { se deben controlar }\end{array}$ & Requisitos cualitativos & Frecuencia de los controles \\
\hline Apariencia & $\begin{array}{l}\text { Ausencia de hemólisis, precipitación, partículas, o formación de gel de- } \\
\text { tectables mediante examen visual }\end{array}$ & Cada nuevo lote \\
\hline Reactividad y especificidad & $\begin{array}{l}\text { Ausencia de hemólisis inmune, formación de rouleaux o fenómeno de } \\
\text { prozona } \\
\text { Reacciones claras con los hematíes portadores del antígeno } \\
\text { correspondiente } \\
\text { Ausencia de reacciones falsas }\end{array}$ & Cada nuevo lote \\
\hline Potencia & $\begin{array}{l}\text { El suero no diluido debe producir una reacción de } 3+\text { a } 4+\text { en medio } \\
\text { salino con hematíes al } 3 \% \text { a temperatura ambiente. Su titulación } \\
\text { debe ser de } 1 / 128 \text { para al anti-A, anti-B, y anti-AB con hematíes } A_{1} \\
\text { y } B \text {, y de } 1 / 64 \text { con hematíes } A_{2} \text { y } A_{2} B\end{array}$ & Cada nuevo lote \\
\hline
\end{tabular}

CUADRO 5. Control de calidad de los reactivos. Sueros $\mathrm{Rh}$

\begin{tabular}{|c|c|c|}
\hline $\begin{array}{l}\text { Parámetros que } \\
\text { se deben controlar }\end{array}$ & Requisitos cualitativos & Frecuencia de los controles \\
\hline Apariencia & $\begin{array}{l}\text { Lo mismo que para los sueros } A B O \\
\text { Lo mismo que para los sueros } A B O\end{array}$ & $\begin{array}{l}\text { Diaria } \\
\text { Diaria }\end{array}$ \\
\hline Reactividad y especificidad & $\begin{array}{l}\text { El suero sin diluir debe dar una reacción de } 3+\text { a } 4+\text { en el test diseñado } \\
\text { para cada suero }\end{array}$ & \\
\hline Potencia & $\begin{array}{l}\text { Titulación de } 1 / 16 \text { para anti-D, anti-C, anti-E, anti-c, anti-e y anti-CDE, } \\
\text { utilizando hematíes } R_{1} r, R_{2} r, r^{\prime} r, o r^{\prime \prime} r\end{array}$ & Cada nuevo lote \\
\hline
\end{tabular}

CUADRO 6. Control de calidad de los reactivos. Suero antiglobulina humana (polivalente)

\begin{tabular}{ccc}
\hline $\begin{array}{c}\text { Parámetros que } \\
\text { se deben controlar }\end{array}$ & Requisitos cualitativos \\
\hline Apariencia & $\begin{array}{c}\text { Ausencia de turbidez, precipitado, partículas o formación de gel en el exa- } \\
\text { men visual }\end{array}$ \\
Reactividad y especificidad & $\begin{array}{c}\text { Ausencia de actividad aglutinante o hemolítica de los hematíes no sensi- } \\
\text { bilizados de cualquier grupo ABO } \\
\text { Aglutinación de hematíes sensibilizados con un suero anti-D que con- } \\
\text { tenga una actividad de anticuerpos }<10 \mathrm{ng} / \mathrm{mL}\end{array}$ \\
$\begin{array}{c}\text { Aglutinación de hematíes sensibilizados por un suero que fije comple- } \\
\text { mento (p.e. anti-Jka ), a un título más elevado en presencia que en } \\
\text { ausencia de complemento } \\
\text { Aglutinación de hematíes recubiertos de C3b y C3d y aglutinación débil o } \\
\text { nula con hematíes recubiertos de C4b y C4d }\end{array}$ & Cada nuevo lote \\
\hline
\end{tabular}

1. La empresa suministradora le proporcionará al técnico la formación necesaria para que sepa usarlo.

2. Para cada aparato habrá una ficha donde se irán anotando todos los incidentes, reparaciones y operaciones de mantenimiento. Se establecerá una vida media después de la cual el aparato se considerará amortizado.

3. En el momento de adquirir un aparato y después de hacerle reparaciones importantes, es necesa- rio someterlo a un control o una calibración (centrífugas, incubadoras de temperatura, lavadora de Coombs, etc.) (cuadro 9).

4. Es preciso planificar un mantenimiento periódico interno, realizado dentro del propio laboratorio, que comprenderá actividades de limpieza y de índole menor, así como un control externo para aquellos aparatos que se consideran críticos para garantizar la validez de los resultados. El control externo será realizado preferentemente 
CUADRO 7. Control de calidad de las técnicas. Tipificación Rh

\begin{tabular}{|c|c|c|c|}
\hline Tipo de prueba & Requisitos mínimos & Muestras de control & Frecuencia de los controles \\
\hline Tipificación del factor Rh (D) & $\begin{array}{l}\text { Usar dos sueros anti-D diferentes } \\
\text { Usar el test indirecto de antiglobu- } \\
\text { lina para detectar los } D^{\mathrm{u}} \text {, si es } \\
\text { necesario } \\
\text { Si se utilizan dos sueros mono- } \\
\text { clonales deben ser de lotes di- } \\
\text { ferentes y capaces de recono- } \\
\text { cer con certeza las variantes } \\
\text { del antígeno D. }\end{array}$ & Una muestra D+ y otra D- & $\begin{array}{l}\text { En cada serie de pruebas, } \\
\text { o al menos una vez al } \\
\text { día, siempre que se usen } \\
\text { los mismos reactivos }\end{array}$ \\
\hline Fenotipo Rh & $\begin{array}{l}\text { Tipificación por duplicado usando } \\
\text { dos sueros para cada antígeno } \\
(\mathrm{C}, \mathrm{c}, \mathrm{E}, \mathrm{e})\end{array}$ & $\begin{array}{l}\text { Para una fenotipificación com- } \\
\text { pleta, una muestra de cada uno } \\
\text { de los siguientes } R h: R_{1} r, R_{2} r \text {, } \\
r^{\prime} r, r^{\prime \prime} r, r \text { y } R_{1}{ }^{w} r \text {. }\end{array}$ & Ídem \\
\hline
\end{tabular}

CUADRO 8. Control de calidad de las técnicas. Investigación de anticuerpos irregulares

\begin{tabular}{|c|c|c|c|}
\hline Tipo de prueba & Requisitos mínimos & Muestras de control & Frecuencia de los controles \\
\hline $\begin{array}{l}\text { Detección de Ac anti-A } \\
\text { y anti-B }\end{array}$ & Usar hematíes $A_{1}$ y $B$ & $\begin{array}{l}\text { Muestras de suero con un título de } \\
\text { Ac anti-A y anti-B superior e in- } \\
\text { ferior al aceptado para anti-A y } \\
\text { anti-B, respectivamente }\end{array}$ & En cada serie de pruebas \\
\hline $\begin{array}{l}\text { Detección de Ac irregulares en } \\
\text { donantes }\end{array}$ & $\begin{array}{l}\text { Usar una prueba que detecte los } \\
\text { Ac clínicamente significativos }\end{array}$ & $\begin{array}{l}\text { Muestras de sueros con Ac } \\
\text { conocidos }\end{array}$ & $\begin{array}{l}\text { Ocasional (introducidos } \\
\text { por el supervisor del } \\
\text { laboratorio) }\end{array}$ \\
\hline Detección de Ac en receptores & $\begin{array}{l}\text { Utilizar como mínimo un test de } \\
\text { antiglobulina indirecto. Si se uti- } \\
\text { lizan otros métodos manuales } \\
\text { o automáticos deben tener una }\end{array}$ & $\begin{array}{l}\text { Muestras de sueros con Ac } \\
\text { conocidos }\end{array}$ & $\begin{array}{l}\text { Ocasional (introducidos } \\
\text { por el supervisor del } \\
\text { laboratorio) }\end{array}$ \\
\hline
\end{tabular}

Ac: anticuerpos.

CUADRO 9. Control de calidad del equipo

\begin{tabular}{lll}
\hline \multicolumn{1}{c}{ Equipo } & \multicolumn{1}{c}{ Control } & \multicolumn{1}{c}{ Frecuencia } \\
\hline $\begin{array}{l}\text { Centrífuga de laboratorio } \\
\text { Centrífuga } \\
\text { Lavadora de Coombs }\end{array}$ & $\begin{array}{l}\text { Cronometrar la velocidad, aceleración y demora } \\
\text { Utilizar hematíes sensibilizados con anti-D }\end{array}$ & $\begin{array}{l}\text { Ocasional } \\
\text { Diaria }\end{array}$ \\
$\begin{array}{l}\text { Refrigeradores } \\
\text { Congeladores }\end{array}$ & Termómetros de precisión & Diaria \\
$\begin{array}{l}\text { Baños termostáticos } \\
\text { Pehachímetros }\end{array}$ & Soluciones de control de pH: $4-7$ y $7-10$ & Cuando se usan \\
\hline
\end{tabular}

por la empresa que lo suministró y puede ser anual o no, según la actividad a la que esté sometido el aparato.

5. En el caso de los sistemas informáticos se establecerán procedimientos de verificación y control.

\section{LA FORMACIÓN DE LOS RECURSOS HUMANOS}

La posesión de los títulos apropiados y la adecuada formación del personal que practica las téc- 
nicas son fundamentales como garantía de los resultados obtenidos. El personal debe saber no solo cómo realizar las tareas encomendadas, sino el porqué de las mismas y las consecuencias de no seguir los procedimientos aprobados. Antes de encomendarle la responsabilidad de una determinada tarea a un miembro del personal, es necesario que este haya superado una formación adaptada a su puesto y algunas pruebas de pericia. Se establecerá una formación continuada breve de forma periódica y una formación específica ante variaciones de las tareas, la aplicación de nuevas técnicas, o cualquier otro cambio. El personal deberá firmar siempre el trabajo que realiza y la acumulación de errores será motivo de formación adicional.

\section{LA AUTOMATIZACIÓN COMO MEDIO PARA ELIMINAR ERRORES}

La automatización ha venido a impartir rapidez, estandarización y seguridad a las pruebas de laboratorio. Siguiendo las indicaciones del fabricante, los procesos automatizados evitan errores en los procedimientos y en la transcripción de datos, siempre que los resultados se trasmitan directamente al ordenador central.

En inmunohematología, las reacciones de aglutinación son captadas por lectores automáticos que utilizan una técnica fotométrica. El sistema informático determina, por ejemplo, el grupo sanguíneo de cada muestra comparando la reacción de aglutinación positiva o negativa de la muestra con las reacciones observadas en sueros usados a manera de patrón. Mediante el uso de etiquetas con códigos de barras se consigue identificar correctamente las muestras y los resultados, proporcionando al técnico una seguridad adicional.

\section{LA ORGANIZACIÓN, INSPECCIÓN Y AUTORIZACIÓN DE CENTROS PARTICULARES COMO GARANTÍA DE LA CALIDAD}

No basta solamente con superar ciertos aspectos técnicos para lograr la seguridad y calidad que se exigen en el campo de la medicina transfusional. Todo grupo de trabajo debe contar con una organización interna en la que todo esté convenientemente regulado y previsto. Las actividades que se realizan y la organización del trabajo deben constar por escrito, así como las responsabilidades de cada miembro del equipo.

Ciertas medidas confieren una garantía de calidad adicional y contribuyen a enriquecer el sis- tema sanitario. Entre ellas figura la autorización concedida a un centro determinado por las autoridades sanitarias, organismos e instituciones responsables para que ejerza las actividades específicas que se solicitan, una vez que aquellas han comprobado que cumple con los requisitos en cuanto a instalaciones adecuadas y bien equipadas, personal idóneo y con los títulos exigidos y la superación de inspecciones periódicas.

\section{CONTROLES DE CALIDAD EXTERNOS}

La participación voluntaria en programas de control de calidad externos confiere a un centro la oportunidad de someter a revisión periódica los resultados que obtiene y de identificar posibles deficiencias que debe superar. Se establece así una evaluación continua.

\section{EL PROGRAMA DE CONTROL DE CALIDAD EXTERNO EN INMUNOHEMATOLOGÍA DE LA OPS Y EL CENTRO DE TRANSFUSIÓN DE LA COMUNIDAD VALENCIANA}

En 1996, la OPS, especialmente interesada en mejorar la seguridad transfusional en inmunohematología en los países de la Región de las Américas, solicitó la colaboración de esta autora para diseñar y establecer un Programa de Control de Calidad Externo en Inmunohematología dirigido a todos los bancos de sangre de los países de la Región que, de forma voluntaria, quisieran participar. Este programa se enmarcó en un plan de actuaciones conjuntas de la OPS, la Agencia Española de Cooperación Internacional y el Ministerio de Sanidad y Consumo de España, y su objetivo ha sido establecer un control semestral de los procedimientos operativos empleados en los laboratorios de inmunohematología participantes, haciendo especial hincapié en las siguientes técnicas:

- Tipificación eritrocitaria de los sistemas de grupo sanguíneo que con más frecuencia se transfunden.

- Escrutinio e identificación de anticuerpos irregulares.

- Prueba directa de antiglobulina.

El objetivo final es que cada centro participante identifique sus deficiencias y planifique su mejoramiento. Para cumplir el objetivo previsto, cada centro participante recibe y analiza, dos veces al año, dos muestras sanguíneas (eritrocitos y suero) elegidas por el coordinador del programa. 
FIGURA 1. Evolución del número de centros participantes

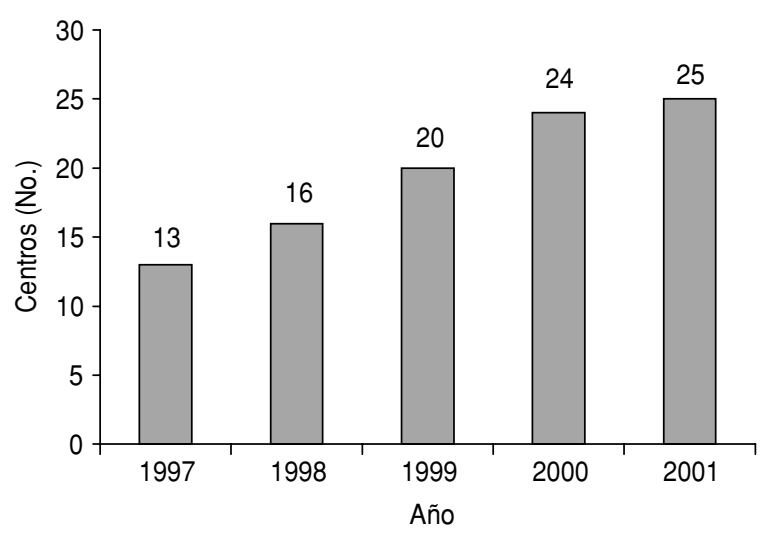

CUADRO 10. Porcentajes de resultados erróneos

\begin{tabular}{lccc}
\hline \multicolumn{1}{c}{ Prueba } & 1998 & 1999 & 2000 \\
\hline $\begin{array}{l}\text { Escrutinio de anticuerpos } \\
\text { irregulares }\end{array}$ & 7,5 & 6,2 & 0 \\
$\begin{array}{l}\text { Identificación de anticuerpos } \\
\quad \text { irregulares }\end{array}$ & 17,5 & 12,5 & 5,2 \\
Prueba de antiglobulina directa & 5 & 0 & 0 \\
Fenotipo Rh & 2,5 & 5,8 & 0 \\
Fenotipo Kell & 5 & 6,2 & 0 \\
\hline
\end{tabular}

\section{METODOLOGÍA}

1. Cada centro participante tiene asignado un código numérico para garantizar la confidencialidad de los resultados.

2. Cada centro participante debe analizar las muestras del control de calidad utilizando sus técnicas habituales, como si fueran parte de las muestras sometidas a tamizaje en la rutina diaria.

3. Mediante impresos adecuados, cada centro participante envía los resultados al Coordinador del Programa.

4. El Coordinador del Programa envía al participante un informe final sobre la idoneidad o falta de ajuste de los resultados obtenidos.

El Programa se inició en 1996 con la participación de 13 bancos de sangre y a finales de 2001 contaba con 25 bancos de sangre en 18 países (figura 1 ).

En el diseño inicial se planificó que en el futuro cada banco de sangre que participara en el Pro- grama pudiera a su vez controlar, mediante un sistema similar, a los demás bancos de sangre de su zona de influencia.

El cuadro 10 muestra la evolución de los errores observados. A lo largo del tiempo se observa no solo un incremento de los resultados correctos, sobre todo en los laboratorios que han participado más tiempo en el Programa, sino que en la actualidad todos los centros participantes realizan todas las pruebas. Anteriormente algunos no realizaban determinadas pruebas debido, casi siempre, a que carecían del reactivo apropiado. En estos años que ha funcionado el Programa, el grupo se ha reunido tres veces para valorarlo y tener la posibilidad de dar instrucción acerca de cómo corregir determinados fallos técnicos.

Si los resultados obtenidos en un programa de control de calidad externo valoran de forma global la idoneidad de las técnicas, los reactivos utilizados y la formación de personal, y si dichos resultados son, sin duda, un magnífico indicador de la calidad del laboratorio participante, la integración voluntaria en estos programas representa el compromiso de la dirección por la calidad asistencial del centro.

\section{SYNOPSIS}

\section{Quality control of immunologic testing of blood in the Region of the Americas}

Assuring the best quality in transfusion medicine necessarily involves exercising exhaustive control over the entire system and developing protocols for procedures and techniques, the reagents and equipment used, personnel training, and so on. Additional guarantees can come from adequately organizing the work and perhaps automating processes in order to avoid errors. Another way to avoid errors in the laboratory is to register and continuously analyze the mistakes that do occur, since that makes it necessary to change procedures in order to avoid repeating them. External quality control programs (EQCP) make it possible to perform a periodic overall assessment of the suitability of techniques, reagents, and training of personnel in relation to the validity of the results obtained. By voluntarily participating in an EQCP, the blood center shows its commitment to the quality of care. In the Region of the Americas, the Pan American Health Organization's Immunohematology Quality Control Program has made it possible to objectively quantify the improvement attained in the formative level reached by participating blood banks in the four years the Program has been in operation. 